\title{
Edge Pair Sum Labeling
}

\author{
P. Jeyanthi ${ }^{1 *}$ and T. Saratha Devi ${ }^{2}$ \\ ${ }^{1}$ Research Centre, Department of Mathematics, Govindammal Aditanar College for Women, \\ Thiruchendur 628 215. Tamilnadu, India
}

${ }^{2}$ Department of Mathematics, G. Venkataswamy Naidu College, Kovilpatti, Tamilnadu, India

Received 18 May 2013, accepted in final revised form 22 August 2013

\begin{abstract}
An injective map $f: E(G) \rightarrow\{ \pm 1, \pm 2, \ldots, \pm q\}$ is said to be an edge pair sum labeling of a graph $G(p, q)$ if the induced vertex function $f^{*}: V(G) \rightarrow Z-\{0\}$ defined by $f^{*}(v)=$ $\sum_{e \in E_{v}} f(e)$ is one - one, where $E_{v}$ denotes the set of edges in $G$ that are incident with a vertex $v$ and $f^{*}(V(G))$ is either of the form $\left\{ \pm k_{1}, \pm k_{2}, \ldots, \pm k \frac{p}{2}\right\}$ or $\left\{ \pm k_{1}, \pm k_{2}, \ldots, \pm k_{\frac{p-1}{2}}\right\} \cup\left\{k \frac{p}{2}\right\}$ according as $p$ is even or odd. A graph with an edge pair sum labeling is called an edge pair sum graph. In this paper we prove that path $P_{n}$, cycle $C_{n}$, triangular snake, $P_{m} \cup K_{1, n}, C_{n} \odot K_{m}^{c}$ are edge pair sum graphs.
\end{abstract}

Keywords: Pair sum graph, edge pair sum labeling, edge pair sum graph.

() 2013 JSR Publications. ISSN: 2070-0237 (Print); 2070-0245 (Online). All rights reserved.

doi: http://dx.doi.org/10.3329/jsr.v5i3.15001 J. Sci. Res. 5 (3), 457-467 (2013)

\section{Introduction}

We consider only finite, simple, planar and undirected graphs. A graph $G(p, q)$ has the vertex-set $V(G)$ and the edge-set $E(G)$ with $|V(G)|=p$ and $|E(G)|=q$. A vertex labeling $f$ of a graph $G$ is an assigned of labels to the vertices of $\mathrm{G}$ that induces a label for each edge xy depending on the vertex labels. An edge labeling $f$ of a graph $G$ is an assigned of labels to the edges of $G$ that induces a label for each vertex $v$ depending on the labels of the edges incident on it. Terms and terminology are used in the sense of Harary [1].

Ponraj and Parthipan [2] introduced the concept of pair sum labeling. An injective map $f: V(G) \rightarrow\{ \pm 1, \pm 2, \ldots, \pm p\}$ is said to be a pair sum labeling of a graph $G(p, q)$ if the induced edge function $f_{e}: E(G) \rightarrow Z$ - $\{0\}$ defined by $f_{e}(u v)=f(u)+f(v)$ is one-one and $f_{e}(E(G))$ is either of the form $\left\{ \pm k_{1}, \pm k_{2}, \ldots, \pm k_{\frac{q}{2}}\right\}$ or $\left\{ \pm k_{1}, \pm k_{2}, \ldots, \pm k_{\frac{q-1}{2}}\right\} \cup\left\{k_{\frac{q+1}{2}}\right\}$

\footnotetext{
*Corresponding author: jeyajeyanthi@ rediffmail.com
} 
according as $q$ is even or odd. A graph with a pair sum labeling is called a pair sum graph. The pair sum behavior of graphs like complete graph, path, bistar, cycle, all trees of order $\leq 8$, and $\leq 9$ and some more standard graphs are investigated in refs. [3-6].

Motivated by Ponraj and Parthipan [2], we define a new labeling called an edge pair sum labeling analogous to pair sum labeling. Let $G(p, q)$ be a graph. An injective map $f$ : $E(G) \rightarrow\{ \pm 1, \pm 2, \ldots, \pm q\}$ is said to be an edge pair sum labeling if the induced vertex function $f^{*}: V(G) \rightarrow Z-\{0\}$ is defined by $f^{*}(v)=\sum_{e \in E_{v}} f(e)$ is one - one where $E_{v}$ denotes the set of edges in $G$ that are incident with a vertex $v$ and $f^{*}(V(G))$ is either of the form $\left\{ \pm k_{1}, \pm k_{2}, \ldots, \pm k_{\frac{p}{2}}\right\}$ or $\left\{ \pm k_{1}, \pm k_{2}, \ldots, \pm k_{\frac{p-1}{2}}\right\} \cup\left\{k_{\frac{p}{2}}\right\}$ according as $\mathrm{p}$ is even or odd. A graph with an edge pair sum labeling is called an edge pair sum graph.

We use the following definitions in the subsequent sequel.

\section{Definition 1.1}

The union of two graphs $G_{1}$ and $G_{2}$ is the graph $G_{1} \cup G_{2}$ with $V\left(G_{1} \cup G_{2}\right)=V\left(G_{1}\right) \cup V\left(G_{2}\right)$ and $E\left(G_{1} \cup G_{2}\right)=E\left(G_{1}\right) \cup E\left(G_{2}\right)$.

\section{Definition 1.2}

The corona $G \odot H$ is the graph obtained by taking one copy of $G$ and $n$ copies of $H$ and joins the $i$ th vertex of $G$ with an edge to every vertex in the $i$ th copy of $H$ where $|V(G)|=$ $n$.

\section{Main results}

Theorem 2.1: Every path $P_{n}$ is an edge pair sum graph for $n \geq 3$.

Proof: Let $V=\left\{v_{1}, v_{2}, \ldots, v_{n}\right\}$ and $E=\left\{e_{1}, e_{2}, \ldots, e_{n-1}\right\}$ be the vertex set and edge set of $P_{n}$ respectively, where $e_{i}=v_{i} v_{i+1}, 1 \leq i \leq n-1$. We consider the following four cases:

Case (i) $n=3$.

Define the labeling $f: E(G) \rightarrow\{ \pm 1, \pm 2\}$ by $f\left(e_{1}\right)=-2, f\left(e_{2}\right)=1$. The induced vertex labeling are $f^{*}\left(v_{1}\right)=-2, f^{*}\left(v_{2}\right)=-1, \quad f^{*}\left(v_{3}\right)=1$. Hence, $f$ is an edge pair sum labeling of $P_{3}$.

Case (ii) $n=4$.

Define the labeling $f: E(G) \rightarrow\{ \pm 1, \pm 2, \pm 3\}$ by $f\left(e_{1}\right)=-2, f\left(e_{2}\right)=-1, f\left(e_{3}\right)=3$. The induced vertex labelings are $f^{*}\left(v_{1}\right)=-2, f^{*}\left(v_{2}\right)=-3, f^{*}\left(v_{3}\right)=2, f^{*}\left(v_{4}\right)=3$. Hence, $f$ is an edge pair sum labeling of $P_{3}$.

Case (iii) $n$ is even. Take $n=2 k, k \geq 3$.

Define the labeling $f: E(G) \rightarrow\{ \pm 1, \pm 2, \ldots, \pm(n-1)\}$ by

$$
f\left(e_{i}\right)= \begin{cases}-2 & \text { if } i=k-1 \\ -1 & \text { if } i=k \\ 3 & \text { if } i=k+1\end{cases}
$$




$$
f\left(e_{i}\right)=\left\{\begin{array}{cl}
2 k+1-2 i & \text { if } 1 \leq i \leq k-2 \\
2 k-1-2 i & \text { if } k+2 \leq i \leq 2 k-1
\end{array}\right.
$$

The induced vertex labelings are

$f^{*}\left(v_{1}\right)=f\left(e_{1}\right)=2 k-1, f^{*}\left(v_{n}\right)=f\left(e_{2 k-1}\right)=-(2 k-1)$, for $2 \leq i \leq k-2$

$f^{*}\left(v_{i}\right)=f\left(e_{i-1}\right)+f\left(e_{i}\right)=4(k+1-i), f^{*}\left(v_{k-1}\right)=3, f^{*}\left(v_{k}\right)=-3, f^{*}\left(v_{k+1}\right)=$

$2, f^{*}\left(v_{k+2}\right)=-2$ and for $k+3 \leq i \leq 2 k-1 f^{*}\left(v_{i}\right)=f\left(e_{i-1}\right)+f\left(e_{i}\right)=4(k-$

i).

From the above vertex labeling we get $f^{*}(V(G))=\{ \pm 2, \pm 3, \pm 12, \pm 16, \ldots, \pm 4(k-$ $1, \pm\left(2 k^{-1}\right)$. Hence, $\mathrm{f}$ is an edge pair sum labeling of $P n$.

Case (iv) $n$ is odd. Take $n=2 k+1, k \geq 2$.

Define the labeling $f: E(G) \rightarrow\{ \pm 1, \pm 2, \ldots, \pm(n-1)\}$ by

$$
\begin{aligned}
& f\left(e_{i}\right)=\left\{\begin{aligned}
1 & \text { if } i=k+1 \\
2 & \text { if } i=k \\
-5 & \text { if } i=k-1
\end{aligned}\right. \\
& f\left(e_{i}\right)=\left\{\begin{array}{lr}
5 & i=k+2 \\
-(2 k+3-2 i) & \text { if } \\
-2 k+1+2 i & \text { if } 1 \leq i \leq k-2 \\
& \text { if } k+3 \leq i \leq 2 k
\end{array}\right.
\end{aligned}
$$

The induced vertex labelings are

$$
\begin{aligned}
& f^{*}\left(v_{1}\right)=f\left(e_{1}\right)=-2 k-1, f^{*}\left(v_{n}\right)=f\left(e_{2 k}\right)=(2 k+1), \\
& \text { for } 2 \leq i \leq k-1 f^{*}\left(v_{i}\right)=f\left(e_{i-1}\right)+f\left(e_{i}\right)=4(-k+i-2), f^{*}\left(v_{k}\right)=-3, \\
& f^{*}\left(v_{k+1}\right)=3, f^{*}\left(v_{k+2}\right)=6 \text { and for } k+3 \leq i \leq 2 k f^{*}\left(v_{i}\right)=f\left(e_{i-1}\right)+f\left(e_{i}\right) \\
& =-4(k-i) .
\end{aligned}
$$

From the above argument we get $f^{*}(V(G))=\{ \pm 3, \pm 12, \pm 16, \ldots, \pm 4 k, \pm(2 k+$ 1)U6. Hence, $f$ is an edge pair sum labeling of $P n$.

Theorem 2.2: Every cycle $C_{n}(n \geq 3)$ is an edge pair sum graph.

Proof : Let $V=\left\{v_{1}, v_{2}, \ldots, v_{n}\right\}$ and $E=\left\{e_{1}, e_{2}, \ldots, e_{n}\right\}$ be the vertex set and the edge set of $C_{n}$, where $e_{i}=v_{i} v_{i+1}, 1 \leq i \leq n-1$ and $e_{n}=v_{n} v_{1}$.

Define the edge labeling $f: E(G) \rightarrow\{ \pm 1, \pm 2, \ldots, \pm n\}$ by considering the following three cases.

Case (i) $n=3$.

$f\left(e_{1}\right)=-1, f\left(e_{2}\right)=2, f\left(e_{3}\right)=-3$. The induced vertex labelings are $f^{*}\left(v_{1}\right)=$ $-4, f^{*}\left(v_{2}\right)=1, f^{*}\left(v_{3}\right)=-1$. Clearly, $f^{*}(V(G))=\{ \pm 1,-4\}$. Hence $f$ is an edge pair sum labeling of $C_{3}$.

Case (ii) $n=4$.

$f\left(e_{1}\right)=1, f\left(e_{2}\right)=2, f\left(e_{3}\right)=-1, f\left(e_{4}\right)=-2$. The induced vertex labelings are $f^{*}\left(v_{1}\right)=-1, \quad f^{*}\left(v_{2}\right)=3, f^{*}\left(v_{3}\right)=1, \quad f^{*}\left(v_{4}\right)=-3 . \quad$ Clearly, $\quad f^{*}(V(G))=$ $\{ \pm 1, \pm 3\}$. Hence $f$ is an edge pair sum labeling of $C_{4}$. 
Case (iii) $n$ is even. Take $n=2 m$.

Subcase (i) $m$ is odd.

For $1 \leq i \leq m \quad f\left(e_{i}\right)=i$ and $f\left(e_{m+i}\right)=-i$. The induced vertex labelings are as follows:

For $1 \leq i \leq m-1 f^{*}\left(v_{i+1}\right)=f\left(e_{i}\right)+f\left(e_{i+1}\right)=(2 i+1), f^{*}\left(v_{m+1}\right)=f\left(e_{m}\right)+$ $f\left(e_{m+1}\right)=(m-1)$,

for $1 \leq i \leq m-1 \quad f^{*}\left(v_{m+1+i}\right)=f\left(e_{m+i}\right)+f\left(e_{m+1+i}\right)=-(2 i+1), \quad f^{*}\left(v_{1}\right)=$ $f\left(e_{2 m}\right)+f\left(e_{1}\right)=-(m-1)$.

Thus we get $f^{*}(V(G))=\{ \pm 3, \pm 5, \pm 7, \ldots, \pm(2 m-1), \pm(m-1)\}$. Hence, $f$ is an edge pair sum labeling.

Subcase (ii) $m$ is even and $m>2$.

For $1 \leq i \leq m f\left(e_{i}\right)=i, f\left(e_{m+1}\right)=-2, f\left(e_{m+2}\right)=-1$,

for $1 \leq i \leq m-2 f\left(e_{m+2+i}\right)=f\left(e_{m+i}\right)-2$.

The induced vertex labelings are as follows:

For $1 \leq i \leq m-1 f^{*}\left(v_{i+1}\right)=f\left(e_{i}\right)+f\left(e_{i+1}\right)=2 i+1, f^{*}\left(v_{m+1}\right)=f\left(e_{m}\right)+$ $f\left(e_{m+1}\right)=m-2$, for $1 \leq i \leq m-1 f^{*}\left(v_{m+1+i}\right)=f\left(e_{m+i}\right)+f\left(e_{m+1+i}\right)=$ $-(2 \mathrm{i}+1), f^{*}\left(v_{1}\right)=f\left(e_{2 m}\right)+f\left(e_{1}\right)=-(m-2)$.

From the above labeling, we get $f^{*}(V(G))=\{ \pm 3, \pm 5, \pm 7, \ldots, \pm(2 m-1), \pm(m-2)\}$. Hence, $\mathrm{f}$ is an edge pair sum labeling.

Case (iv) $n$ is odd. Take $n=2 m+1, m \geq 2$.

Subcase (i) $m \equiv 0,2(\bmod 3)$

$f\left(e_{1}\right)=1, f\left(e_{2}\right)=-2$, for $1 \leq i \leq m-1 f\left(e_{2+i}\right)=2+i, f\left(e_{m+2}\right)=-1$,

for $1 \leq i \leq m-1 f\left(e_{m+2+i}\right)=-(2+i)$.

The induced vertex labelings are

$f^{*}\left(v_{2}\right)=-1, f^{*}\left(v_{3}\right)=1$, for $3 \leq i \leq m f^{*}\left(v_{i+1}\right)=f\left(e_{i}\right)+f\left(e_{i+1}\right)=2 i+1$,

$f^{*}\left(v_{m+2}\right)=m, f^{*}\left(v_{m+3}\right)=-4$, for $3 \leq i \leq m f^{*}\left(v_{m+1+i}\right)=f\left(e_{m+i}\right)+$ $f\left(e_{m+1+i}\right)=-(2 i+1), f^{*}\left(v_{1}\right)=-m$.

From the above arguments, we get $f^{*}(V(G))=\{ \pm 1, \pm 7, \pm 9, \ldots, \pm(2 m+$ $1, \pm m U-4$. Hence, $f$ is an edge pair sum labeling.

Subcase (ii) $m \equiv 1(\bmod 3)$

$f\left(e_{1}\right)=1,\left(e_{2}\right)=2, f\left(e_{3}\right)=-3$, for $1 \leq i \leq m-2 f\left(e_{3+i}\right)=(3+i), f\left(e_{m+2}\right)=$ $-1, f\left(e_{m+3}\right)=-2$, for $1 \leq i \leq m-2 f\left(e_{m+3+i}\right)=-(3+i)$.

The induced vertex labeling are

$f^{*}\left(v_{2}\right)=3, f^{*}\left(v_{3}\right)=-1, f^{*}\left(v_{4}\right)=1$, for $4 \leq i \leq m f^{*}\left(v_{i+1}\right)=f\left(e_{i}\right)+f\left(e_{i+1}\right)$

$=(2 i+1), f^{*}\left(v_{m+2}\right)=m, f^{*}\left(v_{m+3}\right)=-3, f^{*}\left(v_{m+4}\right)=-6$, for $4 \leq i \leq$ $m f^{*}\left(v_{m+1+i}\right)=f\left(e_{m+i}\right)+f\left(e_{m+1+i}\right)=-(2 i+1), f^{*}\left(v_{1}\right)=-m$.

Clearly, $f^{*}(V(G))=\{ \pm 1, \pm 3, \pm 9, \pm 11, \ldots, \pm(2 m+1), \pm m\} \cup\{-6\}$. Hence, $f$ is an edge pair sum labeling.

Theorem 2.3: The star graph $K_{1, n}$ is an edge pair sum graph if and only if $\mathrm{n}$ is even. 
Proof : Let $V\left(K_{1, n}\right)=\left\{v, v_{1}, v_{2}, \ldots, v_{n}\right\}$ and $E\left(K_{1, n}\right)=\left\{e_{1}, e_{2}, e_{3}, \ldots, e_{n}\right\}$ where $e_{i}=v v_{i}$ , $1 \leq \mathrm{i} \leq \mathrm{n}$.

Define $f\left(e_{1}\right)=-1$, for $2 \leq i \leq \frac{n+2}{2} f\left(e_{i}\right)=i$, for $3 \leq i \leq \frac{n+2}{2} f\left(e_{\frac{n-2}{2}+i}\right)=-i$.

The induced vertex labelings are $f^{*}\left(v_{1}\right)=-1$, for $2 \leq i \leq \frac{n+2}{2} f^{*}\left(v_{i}\right)=i$, for $3 \leq i \leq \frac{n+2}{2} \quad f^{*}\left(\frac{v_{n-2}}{2}+i\right)=-i$ and $f^{*}(v)=1$. From the above labelings we get $f^{*}(V(G))=\left\{ \pm 1, \pm 3, \pm 4, \ldots, \pm\left(\frac{n}{2}+1\right)\right\} \cup\{2\}$. Hence, $f$ is an edge pair sum labeling of $K_{1, n}$ if $\mathrm{n}$ is even.

Conversely assume that $\mathrm{n}$ is odd. Let $\mathrm{f}$ be an edge pair sum labeling of $\mathrm{G}$ with labeling $f\left(e_{i}\right)=x_{i}$, for $1 \leq i \leq n$. Then $f^{*}\left(v_{i}\right)=x_{i}$, for $1 \leq i \leq n$ and $f^{*}(v)=\sum_{i=1}^{n} x_{i}$. Since $\mathrm{f}$ is an edge pair sum labeling we have

$$
f^{*}(V(G))=\left\{ \pm k_{1}, \pm k_{2}, \pm k_{3}, \ldots, \pm k_{\frac{n+1}{2}}\right\} .
$$

If $f^{*}(v)=k_{1}$, then there must be a vertex say $v_{1}$ with $f^{*}\left(v_{1}\right)=-k_{1}$. Therefore $\sum x_{i}=-x_{1} \Rightarrow 2 x_{1}+\sum_{i=2}^{n} x_{i}=0$. Since $f$ is an edge pair sum labeling $\sum_{i=2}^{n} x_{i}=0$. Hence $x_{1}=0$ which is a contradiction. Therefore $f$ is not an edge pair sum labeling of $K_{1, n}$ if $\mathrm{n}$ is odd.

Theorem 2.4: The complete graph $K_{4}$ is not an edge pair sum graph.

Proof: Let $\mathrm{f}$ be an edge pair sum labeling of $K_{4}$ with $f\left(e_{1}\right)=x_{1}, f\left(e_{2}\right)=x_{2}, f\left(e_{3}\right)=$ $x_{3}, f\left(e_{4}\right)=x_{4}, f\left(e_{5}\right)=x_{5}, f\left(e_{6}\right)=x_{6}$. Hence the induced vertex labeling are $f^{*}\left(v_{1}\right)=x_{1}+x_{2}+x_{6}, f^{*}\left(v_{2}\right)=x_{1}+x_{4}+x_{5}, f^{*}\left(v_{3}\right)=x_{3}+x_{4}+x_{6}$ and $f^{*}\left(v_{4}\right)=$ $x_{2}+x_{3}+x_{5}$.

Suppose that $f^{*}\left(v_{1}\right)=k_{1}$ then the other vertex labels must be $f^{*}\left(v_{2}\right)=-k_{1}$, $f^{*}\left(v_{3}\right)=k_{2}, f^{*}\left(v_{4}\right)=-k_{2}$. Hence $2 x_{1}+x_{2}+x_{4}+x_{5}+x_{6}=0$ and $x_{2}+2 x_{3}+x_{4}+$ $x_{5}+x_{6}=0$ which implies that $2 x_{1}-2 x_{3}=0$ and hence $x_{1}=x_{3}$, is a contradiction. Therefore, $K_{4}$ is not an edge pair sum graph.

\section{Remark:}

By Theorem 2.1, $K_{1}$ and $K_{2}$ are not edge pair sum graphs. By theorem 2.2, $K_{3}$ is an edge pair sum graph.

Theorem 2.5: The graph $P_{m} \cup K_{1, n}$ is an edge pair sum graph if $\mathrm{m}$ is odd.

Proof : Let $V\left(P_{m}\right)=\left\{u_{1}, u_{2}, \ldots, u_{m}\right\}, E\left(P_{m}\right)=\left\{e_{i}=u_{i} u_{i+1} ; 1 \leq i \leq m-1\right\}, V\left(K_{1, n}\right)=$ $\left\{v, v_{i} ; 1 \leq i \leq n\right\}$ and $E\left(K_{1, n}\right)=\left\{e_{i}^{\prime}=v v_{i} ; 1 \leq i \leq n\right\}$.

Define the edge labeling $f: E\left(P_{m} \cup K_{1, n}\right) \rightarrow\{ \pm 1, \pm 2, \pm 3, \ldots, \pm(m+n-1)\}$ by considering the following six cases.

Case (i) $m=3$ and $n$ is even.

$$
\begin{aligned}
& f\left(e_{1}\right)=1, f\left(e_{2}\right)=2, \text { for } 1 \leq i \leq 2 f\left(e_{i}^{\prime}\right)=-i, \text { for } 1 \leq i \leq \frac{n-2}{2} f\left(e_{2+i}^{\prime}\right)= \\
& -(3+i) \text { and } f\left(e_{\frac{n+2}{2}+i}^{\prime}\right)=(3+i) .
\end{aligned}
$$

The induced vertex labelings are 
$f^{*}\left(u_{1}\right)=1, f^{*}\left(u_{2}\right)=3, f^{*}\left(u_{3}\right)=2, f^{*}\left(v_{1}\right)=-1, f^{*}\left(v_{2}\right)=-2$, for $1 \leq i \leq \frac{n-2}{2}$ $f^{*}\left(v_{2+i}\right)=-(3+i)$ and $f^{*}\left(v_{\frac{n+2}{2}+i}\right)=(3+i)$, for $1 \leq i \leq n f^{*}(v)=$ $\sum f^{*}\left(e_{i}\right)=-3$. Hence we get $f^{*}(V(G))=\left\{ \pm 1, \pm 2, \pm 3, \ldots, \pm\left(\frac{n+4}{2}\right)\right\}$. Thus, $\mathrm{f}$ is an edge pair sum labeling.

Case (ii) $\mathrm{m}=3$ and $\mathrm{n}$ is odd.

$f\left(e_{1}\right)=1, f\left(e_{2}\right)=2$, for $1 \leq i \leq 3 f\left(e_{i}^{\prime}\right)=-i$, for $1 \leq i \leq \frac{n-3}{2} f\left(e_{3+i}^{\prime}\right)=$ $-(6+i)$ and $f\left(e_{\frac{n+3}{2}+i}^{\prime}\right)=6+i$.

The induced vertex labelings are $f^{*}\left(u_{1}\right)=1, f^{*}\left(u_{2}\right)=3, f^{*}\left(u_{3}\right)=2$, for $1 \leq i \leq$ $3 f^{*}\left(v_{i}\right)=-i$, for $1 \leq i \leq \frac{n-3}{2} f^{*}\left(v_{3+i}\right)=-(6+i)$ and $f^{*}\left(\frac{v_{n+3}}{2}+i\right)=(6+i)$, for $1 \leq i \leq n \quad f^{*}(v)=\sum f^{*}\left(e_{i}\right)=-6$. The vertex labeling becomes $f^{*}(V(G))=$ $\left\{ \pm 1, \pm 2, \pm 3, \pm 7, \pm 8, \pm 9, \ldots, \pm\left(\frac{n+9}{2}\right)\right\} \cup\{-6\}$. Hence, $f$ is an edge pair sum labeling.

Case (iii) $m=5,7,9$ and $n$ is even.

For $1 \leq i \leq \frac{m-3}{2} f\left(e_{i}\right)=(2 i-1), f\left(\frac{e_{\frac{m-1}{2}}}{2}=2, f\left(e_{\frac{m+1}{2}}\right)=f\left(e_{\frac{m-3}{2}}\right)+2\right.$, for $1 \leq i \leq \frac{m-3}{2} f\left(e_{\frac{m+1}{2}+i}\right)=-f\left(e_{\frac{m-1}{2}-i}\right), f\left(e_{1}^{\prime}\right)=-2, f\left(e_{2}^{\prime}\right)=-m+2$, for $1 \leq i \leq$ $\frac{n-2}{2} f\left(e_{2+i}^{\prime}\right)=(m+i)$ and $f\left(e_{\frac{n+2}{2}+i}^{\prime}\right)=-(m+i)$.

The induced vertex labelings are

$f^{*}\left(u_{1}\right)=1, f^{*}\left(u_{m}\right)=-1$, for $1 \leq i \leq \frac{m-5}{2} f^{*}\left(u_{1+i}\right)=4 i, f^{*}\left(u_{\frac{m-1}{2}}\right)=(m-2)$, $f^{*}\left(u_{\frac{m+1}{2}}\right)=m, f^{*}\left(u_{\frac{m+3}{2}}\right)=2$, for $1 \leq i \leq \frac{m-5}{2} f^{*}\left(u_{\frac{m+3}{2}+i}\right)=-f^{*}\left(u_{\frac{m-1}{2}-i}\right)$, $f^{*}\left(v_{1}\right)=-2, f^{*}\left(v_{2}\right)=-(m-2)$, for $1 \leq i \leq \frac{n-2}{2} f^{*}\left(v_{2+i}\right)=(m+i)$ and $f^{*}\left(\frac{v_{n+2}}{2}+i\right)=-(m+i)$, for $1 \leq i \leq n f^{*}(v)=\sum f^{*}\left(e_{i}\right)=-m$.

From the above arguments we get $f^{*}(V(G))=\left\{ \pm 1, \pm 2, \pm 4, \pm 8, \ldots, \pm 4\left(\frac{m-5}{2}\right), \pm(m-2), \pm m, \pm(m+1), \pm(m+\right.$ $2, \ldots, \pm(m+n-22)$. Hence, $\mathrm{f}$ is an edge pair sum labeling.

Case (iv) $m=5,7,9$ and $n$ is odd.

Subcase (i) Let $\mathrm{n} \leq 2(\mathrm{~m}-1)$.

For $1 \leq i \leq \frac{m-3}{2} f\left(e_{i}\right)=(2 i-1), f\left(e_{\frac{m-1}{2}}\right)=2, f\left(e_{\frac{m+1}{2}}\right)=f\left(e_{\frac{m-3}{2}}\right)+2$, for $1 \leq i \leq \frac{m-3}{2} f\left(e_{\frac{m+1}{2}+i}\right)=-f\left(e_{\frac{m-1}{2}-i}\right), f\left(e_{1}^{\prime}\right)=-2, f\left(e_{2}^{\prime}\right)=-m+2, f\left(e_{3}^{\prime}\right)=$ $-m$, for $1 \leq i \leq \frac{n-3}{2} f\left(e_{3+i}^{\prime}\right)=(m+i)$ and $f\left(e_{\frac{n+3}{2}+i}^{\prime}\right)=-(m+i)$.

The induced vertex labelings are 
$f^{*}\left(u_{1}\right)=1, f^{*}\left(u_{m}\right)=-1$, for $1 \leq i \leq \frac{m-5}{2} f^{*}\left(u_{1+i}\right)=4 i, f^{*}\left(u_{\frac{m-1}{2}}\right)=(m-2)$,

$f^{*}\left(u_{\frac{m+1}{2}}\right)=m, f^{*}\left(u_{\frac{m+3}{2}}\right)=2$, for $1 \leq i \leq \frac{m-5}{2} f^{*}\left(u_{\frac{m+3}{2}+i}\right)=-f^{*}\left(u_{\frac{m-1}{2}-i}\right)$, $f^{*}\left(v_{1}\right)=-2, f^{*}\left(v_{2}\right)=-(m-2), f^{*}\left(v_{3}\right)=-m$, for $1 \leq i \leq \frac{n-3}{2} \quad f^{*}\left(v_{3+i}\right)=$ $(m+i)$ and $f^{*}\left(v_{\frac{n+3}{2}+i}\right)=-(m+i)$, for $1 \leq i \leq n f^{*}(v)=\sum f^{*}\left(e_{i}\right)=-2 m$.

The vertex labeling becomes $f^{*}(V(G))=\left\{ \pm 1, \pm 2, \pm 4, \pm 8, \ldots, \pm 4\left(\frac{m-5}{2}\right), \pm(m-\right.$ $2, \pm m, \pm m+1, \ldots, \pm(2 m+n-32) \cup-2 m$. Hence, $f$ is an edge pair sum labeling.

Subcase (ii) Let $n>2(m-1)$.

For $1 \leq i \leq \frac{m-3}{2} \quad f\left(e_{i}\right)=(2 i-1), f\left(\frac{e-1-1}{2}\right)=2, f\left(e_{\frac{m+1}{2}}\right)=f\left(e_{\frac{m-3}{2}}\right)+2$, for $1 \leq i \leq \frac{m-3}{2} f\left(e_{\frac{m+1}{2}+i}\right)=-f\left(e_{\frac{m-1}{2}-i}\right), f\left(e_{1}^{\prime}\right)=-2, f\left(e_{2}^{\prime}\right)=-m+2$, $f\left(e_{3}^{\prime}\right)=-m$, for $1 \leq i \leq \frac{n-3}{2} f\left(e_{3+i}^{\prime}\right)=(2 m+i)$ and $f\left(\frac{e_{n+3}^{\prime}}{2}+i\right)=-(2 m+i)$.

The induced vertex labelings are

$f^{*}\left(u_{1}\right)=1, f^{*}\left(u_{m}\right)=-1$, for $1 \leq i \leq \frac{m-5}{2} f^{*}\left(u_{1+i}\right)=4 i, f^{*}\left(\frac{u_{m-1}}{2}\right)=(m-2)$, $f^{*}\left(u_{\frac{m+1}{2}}\right)=m, f^{*}\left(u_{\frac{m+3}{2}}\right)=2$, for $1 \leq i \leq \frac{m-5}{2} \quad f^{*}\left(\frac{u_{m+3}}{2}+i\right)=-f^{*}\left(\frac{u_{\frac{m-1}{2}}-i}{}\right)$ $, f^{*}\left(v_{1}\right)=-2, f^{*}\left(v_{2}\right)=-(m-2), f^{*}\left(v_{3}\right)=-m$, for $1 \leq i \leq \frac{n-3}{2} f^{*}\left(v_{3+i}\right)=$ $(2 m+i)$ and $f^{*}\left(v_{\frac{n+3}{2}+i}\right)=-(2 m+i)$, for $1 \leq i \leq n f^{*}(v)=\sum f\left(e_{i}\right)=-2 m$. From the above labelings we get

$f^{*}(V(G))=\left\{ \pm 1, \pm 2, \pm 4, \pm 8, \ldots, \pm 4\left(\frac{m-5}{2}\right), \pm(m-2), \pm m, \pm(2 m+1), \pm(2 m+\right.$ $2, \ldots, \pm(4 m+n-32) \cup-2 m$.

Hence, $f$ is an edge pair sum labeling.

Case (v) $m \geq 11$ and $n$ is even.

Subcase (i) Let $m<n$.

For $1 \leq i \leq \frac{m-3}{2} f\left(e_{i}\right)=(2 i-1), f\left(\frac{e^{m-1}}{2}\right)=2, f\left(e_{\frac{m+1}{2}}\right)=f\left(e_{\frac{m-3}{2}}\right)+2$, for $1 \leq i \leq \frac{m-3}{2} f\left(\frac{e_{\frac{m+1}{2}}+i}{2}\right)=-f\left(e_{\frac{m-1}{2}-i}\right), f\left(e_{1}^{\prime}\right)=-2, f\left(e_{2}^{\prime}\right)=-m+2$, for $1 \leq i \leq \frac{n-2}{2} f\left(e_{2+i}^{\prime}\right)=(2 m-10+i)$ and $f\left(e_{\frac{n+2}{2}+i}^{\prime}\right)=-(2 m-10+i)$.

The induced vertex labelings are $f^{*}\left(u_{1}\right)=1, f^{*}\left(u_{m}\right)=-1$, for $1 \leq i \leq \frac{m-5}{2} f^{*}\left(u_{1+i}\right)=4 i, f^{*}\left(u_{\frac{m-1}{2}}\right)=(m-2)$,
$f^{*}\left(u_{\frac{m+1}{2}}\right)=m, f^{*}\left(u_{\frac{m+3}{2}}\right)=2$, for $1 \leq i \leq \frac{m-5}{2} f^{*}\left(\frac{u_{\frac{m+3}{2}+i}}{2}\right)=-f^{*}\left(u_{\frac{m-1}{2}-i}\right)$, 
$f^{*}\left(v_{1}\right)=-2, f^{*}\left(v_{2}\right)=-(m-2)$, for $1 \leq i \leq \frac{n-2}{2} f^{*}\left(v_{2+i}\right)=(2 m-10+i)$ and $f^{*}\left(\frac{v_{n+2}}{2}+i\right)=-(2 m-10+i)$, for $1 \leq i \leq n f^{*}(v)=\sum f\left(e_{i}\right)=-m$.

From the above labeling we get

$f^{*}(V(G))=\left\{ \pm 1, \pm 2, \pm 4, \pm 8, \ldots, \pm 4\left(\frac{m-5}{2}\right), \pm(m-2), \pm m, \pm(2 m-9), \pm(2 m-\right.$ $8, \ldots, \pm(4 m+n-222)$. Hence, $\mathrm{f}$ is an edge pair sum labeling.

Subcase (ii) Let $m>n$.

For $1 \leq i \leq \frac{m-3}{2} f\left(e_{i}\right)=(2 i-1), f\left(\frac{e^{\frac{m-1}{2}}}{2}\right)=2, f\left(e_{\frac{m+1}{2}}\right)=f\left(e_{\frac{m-3}{2}}\right)+2$, for $1 \leq i \leq \frac{m-3}{2} f\left(e_{\frac{m+1}{2}+i}\right)=-f\left(e_{\frac{m-1}{2}-i}\right), f\left(e_{1}^{\prime}\right)=-2, f\left(e_{2}^{\prime}\right)=-m+2$, for $1 \leq i \leq \frac{n-2}{2} f\left(e_{2+i}^{\prime}\right)=(2+4 i)$ and $f\left(\frac{e_{n+2}^{\prime}}{2}+i\right)=-(2+4 i)$.

The induced vertex labeling are

$$
\begin{aligned}
& f^{*}\left(u_{1}\right)=1, f^{*}\left(u_{m}\right)=-1, \text { for } 1 \leq i \leq \frac{m-5}{2} f^{*}\left(u_{1+i}\right)=4 i, \\
& f^{*}\left(u_{\frac{m-1}{2}}\right)=(m-2), f^{*}\left(\frac{u_{m+1}}{2}\right)=m, f^{*}\left(\frac{u m+3}{2}\right)=2, \text { for } 1 \leq i \leq \frac{m-5}{2} \\
& f^{*}\left(u_{\frac{m+3}{2}+i}\right)=-f^{*}\left(u_{\frac{m-1}{2}-i}\right), f^{*}\left(v_{1}\right)=-2, f^{*}\left(v_{2}\right)=-(m-2), \text { for } 1 \leq i \leq \frac{n-2}{2} \\
& f^{*}\left(v_{2+i}\right)=(2+4 i) \text { and } f^{*}\left(v_{\frac{n+2}{2}+i}\right)=-(2+4 i), \text { for } 1 \leq i \leq n f^{*}(v)= \\
& \sum f^{*}\left(e_{i}\right)=-m . \text { Thus we get } \\
& f^{*}(V(G))=\left\{ \pm 1, \pm 2, \pm 4, \pm 8, \ldots, \pm 4\left(\frac{m-5}{2}\right), \pm(m-2), \pm m, \pm 6, \pm 10, \ldots, \pm(2 n-\right.
\end{aligned}
$$

2). Hence, $f$ is an edge pair sum labeling.

Case (vi) $m \geq 11$ and $n$ is odd.

Subcase (i) Let $m \geq n$.

For $1 \leq i \leq \frac{m-3}{2} f\left(e_{i}\right)=(2 i-1), f\left(e_{\frac{m-1}{2}}\right)=2, f\left(e_{\frac{m+1}{2}}\right)=f\left(e_{\frac{m-3}{2}}\right)+2$, for $1 \leq i \leq \frac{m-3}{2} f\left(e_{\frac{m+1}{2}+i}\right)=-f\left(e_{\frac{m-1}{2}-i}\right), f\left(e_{1}^{\prime}\right)=-2, f\left(e_{2}^{\prime}\right)=-m+2$, $f\left(e_{3}^{\prime}\right)=-m$, for $1 \leq i \leq \frac{n-3}{2} f\left(e_{3+i}^{\prime}\right)=(2+4 i)$ and $f\left(e_{\frac{n+3}{2}+i}^{\prime}\right)=-(2+4 i)$.

The induced vertex labelings are

$$
\begin{aligned}
& f^{*}\left(u_{1}\right)=1, f^{*}\left(u_{m}\right)=-1, \text { for } 1 \leq i \leq \frac{m-5}{2} f^{*}\left(u_{1+i}\right)=4 i \\
& f^{*}\left(u_{\frac{m-1}{2}}\right)=(m-2), f^{*}\left(u_{\frac{m+1}{2}}\right)=m, f^{*}\left(u_{\frac{m+3}{2}}\right)=2, \text { for } 1 \leq i \leq \frac{m-5}{2} \\
& f^{*}\left(u_{\frac{m+3}{2}+i}\right)=-f^{*}\left(u_{\frac{m-1}{2}-i}\right), f^{*}\left(v_{1}\right)=-2, f^{*}\left(v_{2}\right)=-(m-2), f^{*}\left(v_{3}\right)=-m,
\end{aligned}
$$


for $1 \leq i \leq \frac{n-3}{2} f^{*}\left(v_{3+i}\right)=(2+4 i)$ and $f^{*}\left(v_{\frac{n+3}{2}+i}\right)=-(2+4 i)$, for $1 \leq i \leq$ $n f^{*}(v)=\sum f\left(e_{i}\right)=-2 m$.

From the above arguments we get $f^{*}(V(G))=\left\{ \pm 1, \pm 2 \pm 4, \pm 8, \ldots . . \pm 4\left(\frac{m-5}{2}\right), \pm(m-2), \pm m, \pm 6, \pm 10, \ldots, \pm(2 n-\right.$ 4) $U-2 m$. Hence, $f$ is an edge pair sum labeling.

Subcase (ii) Let $m<n$.

For $1 \leq i \leq \frac{m-3}{2} f\left(e_{i}\right)=(2 i-1), f\left(\frac{e^{m-1}}{2}\right)=2, f\left(e_{\frac{m+1}{2}}\right)=f\left(e_{\frac{m-3}{2}}\right)+2$, for $1 \leq i \leq \frac{m-3}{2} f\left(e_{\frac{m+1}{2}+i}\right)=-f\left(e_{\frac{m-1}{2}-i}\right), f\left(e_{1}^{\prime}\right)=-2, f\left(e_{2}^{\prime}\right)=-m+2, f\left(e_{3}^{\prime}\right)=$ $-m$, for $1 \leq i \leq \frac{m-3}{2} f\left(e_{3+i}^{\prime}\right)=(2 m-10+i)$ and $f\left(e_{\frac{m+3}{2}+i}^{\prime}\right)=-(2 m-10+i)$, for $1 \leq i \leq \frac{n-m}{2} f\left(e_{m+i}^{\prime}\right)=(2 m+i)$ and $f\left(\frac{e_{m+n}^{\prime}}{2}+i\right)=-(2 m+i)$.

The induced vertex labelings are

$$
\begin{aligned}
& f^{*}\left(u_{1}\right)=1, f^{*}\left(u_{m}\right)=-1, \text { for } 1 \leq i \leq \frac{m-5}{2} f^{*}\left(u_{1+i}\right)=4 i, f^{*}\left(u_{\frac{m-1}{2}}\right)=(m-2), \\
& f^{*}\left(u_{\frac{m+1}{2}}\right)=m, f^{*}\left(u_{\frac{m+3}{2}}\right)=2 \text {, for } 1 \leq i \leq \frac{m-5}{2} f^{*}\left(u_{\frac{m+3}{2}+i}\right)=-f^{*}\left(u_{\frac{m-1}{2}-i}\right) \text {, } \\
& f^{*}\left(v_{1}\right)=-2, f^{*}\left(v_{2}\right)=-(m-2), f^{*}\left(v_{3}\right)=-m \text {, for } 1 \leq i \leq \frac{m-3}{2} f^{*}\left(v_{3+i}\right)= \\
& (2 m-10+i) \text { and } f^{*}\left(v_{\frac{m+3}{2}+i}\right)=-(2 m-10+i), \quad \text { for } 1 \leq i \leq \frac{n-m}{2} f^{*}\left(v_{m+i}\right)= \\
& (2 m+i) \text { and } f^{*}\left(\frac{v_{m+n}}{2}+i\right)=-(2 m+i), \text { for } 1 \leq i \leq n f^{*}(v)=\sum f\left(e_{i}\right)=-2 m \text {. }
\end{aligned}
$$

From the above arguments we get

$$
\begin{aligned}
& f^{*}(V(G))=\left\{ \pm 1, \pm 2, \pm 4, \pm 8, \ldots, \pm 4\left(\frac{m-5}{2}\right), \pm(m-2), \pm m, \pm(2 m+1), \pm(2 m+\right. \\
& 2, \ldots, \pm(3 m+n 2) \pm 2 m-9, \pm(2 m-8, \ldots, \pm 5 m-232,-2 m
\end{aligned}
$$

Hence, $f$ is an edge pair sum label

Theorem 2.6: Let $G(p, q)$ is an edge pair sum graph . Then $G \odot K_{n}^{c}$ is also an edge pair sum graph if $\mathrm{n}$ is even.

Proof: Let $\mathrm{f}$ be an edge pair sum labeling of G. Then $f^{*}(V(G))=\left\{ \pm k_{1}, \pm k_{2}, \ldots, \pm k \frac{p}{2}\right\}$ if $\mathrm{p}$ is even.

$$
f^{*}(V(G))=\left\{ \pm k_{1}, \pm k_{2}, \ldots \ldots, \pm k_{\frac{p-1}{2}}\right\} \cup\left\{k \frac{p}{2}\right\} \text { if } \mathrm{p} \text { is odd. }
$$

Let the edge set of $G \odot K_{n}^{c}$ be $E\left(G \odot K_{n}^{c}\right)=E(G) \cup\left\{e_{i j} ; 1 \leq i \leq p\right.$ and $\left.1 \leq j \leq n\right\}$ and the vertex be $V\left(G \odot K_{n}^{c}\right)=V(G) \cup\left\{\mathrm{v}_{\mathrm{ij}}: 1 \leq i \leq p\right.$ and $\left.1 \leq j \leq n\right\}$.

Here $\left|E\left(G \odot K_{n}^{c}\right)\right|=q+n p$. Take $k=\frac{n}{2}$. 
Define $h: E\left(G \cdot K_{n}^{c}\right) \rightarrow\{ \pm 1, \pm 2, \pm 3, \ldots, \pm(q+p n)\}$.

$\mathrm{h}(\mathrm{e})=\mathrm{f}(\mathrm{e})$ if $\mathrm{e} \in \mathrm{E}(\mathrm{G})$
$h\left(e_{i j}\right)=q+k(i-1)+j$
$1 \leq i \leq p, 1 \leq j \leq k$,
$h\left(e_{i(k+j)}\right)=-(q+$
$k i-1+j$
$1 \leq i \leq p, 1 \leq j \leq k$.

The induced vertex labeling are

$$
\begin{array}{ll}
h^{*}\left(v_{i j}\right)=q+k(i-1)+j & 1 \leq i \leq p, 1 \leq j \leq k, \\
h^{*}\left(v_{i(k+j)}\right)=-(q+k(i-1)+j) & 1 \leq i \leq p, 1 \leq j \leq k .
\end{array}
$$

Then $h^{*}\left(V\left(G \odot K_{n}^{c}\right)\right)=\left\{ \pm k_{1}, \pm k_{2}, \ldots, \pm k_{\frac{p}{2}}\right\}$ if $p$ is even.

$h^{*}\left(V\left(G \odot K_{n}^{c}\right)\right)=\left\{ \pm k_{1}, \pm k_{2}, \ldots, \pm \frac{k_{p-1}}{2}\right\} \cup\left\{k \frac{p}{2}\right\}$ if $p$ is odd. Hence, $h$ is an edge pair sum labeling.

Corollary 2.7 : The graph $C_{n} \odot K_{m}^{c}$ is an edge pair sum graph.

Proof: By Theorem 2.6, $C_{n} \odot K_{m}^{c}$ is an edge pair sum graph if $\mathrm{m}$ is even. Let $\mathrm{m}$ is odd and take $=\frac{m-1}{2}$.

The vertex set $V\left(C_{n} \odot K_{m}^{c}\right)=V_{1} \cup V_{2}$, where $V_{1}=\left\{v_{i}: 1 \leq i \leq n\right\}$ and $V_{2}=$ $\left\{v_{i j}: 1 \leq i \leq n\right.$ and $\left.1 \leq j \leq m\right\}$ and the edge set $E\left(C_{n} \odot K_{m}^{c}\right)=\left\{\left\{e_{i}=v_{i} v_{i+1}: 1 \leq\right.\right.$ $i \leq n-1$, en =vnv1 1 eij $: 1 \leq i \leq n$ and $1 \leq j \leq m$.

Define $h: E\left(C_{n} \odot K_{m}^{c}\right)=\{ \pm 1, \pm 2, \pm 3, \ldots \ldots . \pm(n+n m)\}$.

$$
\begin{array}{lll}
h\left(e_{i}\right)=i & 1 \leq i \leq n, \\
h\left(e_{i j}\right)=-i \quad j=1, \quad 1 \leq i \leq n, & \\
h\left(e_{i j}\right)=n+k(i-1)+j-1 & 1 \leq i \leq n, \quad 2 \leq j \leq \\
h\left(e_{i k+j}\right)=-(n+k(i-1)+j-1) \quad 1 \leq i \leq n, & 2 \leq j \leq \frac{m+1}{2} .
\end{array}
$$

The induced vertex labels are as follows:

$$
\begin{array}{lc}
h\left(v_{i}\right)=i & 1 \leq i \leq n, \\
h\left(v_{i j}\right)=-i & j=1, \quad 1 \leq i \leq n, \\
h\left(v_{i j}\right)=n+k(i-1)+j-1 & 1 \leq i \leq n, \quad 2 \leq j \leq \frac{m+1}{2} \\
h\left(v_{i k+j}\right)=-(n+k(i-1)+j-1) & 1 \leq i \leq n, \quad 2 \leq j \leq \frac{m+1}{2} \\
h\left(V\left(C_{n} \odot K_{m}^{c}\right)=\{ \pm 1, \pm 2, \ldots, \pm p, \pm(n+1), \pm(n+2), \ldots, \pm(n+k(n-1)+\right. \\
m-12 .
\end{array}
$$

Hence, $C_{n} \odot K_{m}^{c}$ is an edge pair sum graph.

Observation 2.7: If $\mathrm{G}(\mathrm{p}, \mathrm{q})$ is $\mathrm{r}$ - regular edge pair sum graph with even number of vertices then $\sum_{v \in V} f^{*}(v) d(v)=0$.

Proof: Let $\mathrm{f}$ be an edge pair sum labeling of $\mathrm{G}$. Then $f^{*}(V(G))=\left\{ \pm k_{1}, \pm k_{2}, \ldots, \pm k_{\underline{p}}\right\}$ 
$\sum_{v \in V} f^{*}(v) d(v)=r\left(\sum_{v \in V} f^{*}(v)\right)=0$.

\section{References}

1. F. Harary, Graph Theory, (Narosa Publishing House, New Delhi, 1998). PMid:9929248 PMCid:PMC2232068

2. R. Ponraj and J. V. X. Parthipan, J. Indian Acad. Math. 32 (2) (2010).

3. R. Ponraj, J. V. X. Parthipan, and R. Kala, Intern. J. Math. Combin. 4, (2010).

4. R. Ponraj, J. V. X. Parthipan, and R. Kala, J. Sci. Res. 3 (2), 321 (2011). http://dx.doi.org/10.3329/jsr.v3i2.6290

5. R. Ponraj, J. V. X. Parthipan, Appl. Math. 2, (2011).

6. R. Ponraj, J. V. X. Parthipan and R. Kala, J. Math. Comput. Sci. 2, (2012). 